\title{
Transcriptional regulation of the virulence genes and the biofilm formation associated operons in Vibrio parahaemolyticus
}

Yiquan Zhang ${ }^{1,2^{*}+}{ }^{0}$, Yue Qiu ${ }^{2+}$, Xingfan Xue ${ }^{2}$, Miaomiao Zhang ${ }^{2}$, Junfang Sun ${ }^{4}$, Xue Li ${ }^{4}$, Lingfei Hu ${ }^{3}$, Zhe Yin ${ }^{3}$, Wenhui Yang ${ }^{3}$, Renfei Lu ${ }^{4^{*}}$ and Dongsheng Zhou ${ }^{3^{*}}$

\begin{abstract}
Background: The membrane fusion protein ( $\mathrm{mfp}$ ) gene locus of Vibrio parahaemolyticus consists of two operons, cpsQ-mfpABC and $m f p A B C$, which are both required for biofilm formation. ToxR and CalR are required for the full virulence of $V$. parahaemolyticus, and their mutual regulation has been demonstrated. Moreover, cell density-dependent expression of toxR was previously observed in V. parahaemolyticus, but details about the related mechanisms remained unclear. QsvR can work with the master quorum sensing (QS) regulators AphA and OpaR to regulate virulence expression and biofilm formation.
\end{abstract}

Results: In the present work, we showed that QsvR bound to the promoter-proximal DNA regions of toxR and calR to repress their transcription as well as occupying the regulatory regions of $c p s Q-m f p A B C$ and $m f p A B C$ to activate their transcription. Thus, we reconstructed the QsvR-dependent promoter organization of toxR, calR, cpsQ-mfpABC, and mfpABC.

Conclusion: QsvR directly repressed toxR and calR transcription as well as directly activated cpsQ-mfpABC and $m f p A B C$ transcription. The data presented here promotes us to gain deeper knowledge of the regulatory network of the mfp locus in V. parahaemolyticus.

Keywords: Vibrio parahaemolyticus, QsvR, ToxR, CalR, $m f p$

\section{Background}

Vibrio parahaemolyticus, a Gram-negative halophilic bacterium, naturally inhabits coastal ecosystems and can cause human illness via consumption of raw or undercooked seafood or, less commonly, through small open wounds exposed to seawater [1]. The major clinical

\footnotetext{
*Correspondence: zhangyiquanq@163.com; rainman78@163.com; dongshengzhou1977@gmail.com

${ }^{\dagger}$ Yiquan Zhang and Yue Qiu are considered as co-first authors

${ }^{2}$ School of Medicine, Jiangsu University, Zhenjiang 212013, Jiangsu, China

${ }^{3}$ State Key Laboratory of Pathogen and Biosecurity, Beijing Institute of Microbiology and Epidemiology, Beijing 100071, China

${ }^{4}$ Department of Clinical Laboratory, Nantong Third Hospital Affiliated to Nantong University, Nantong 212006, Jiangsu, China

Full list of author information is available at the end of the article
}

symptoms of $V$. parahaemolyticus infection include watery diarrhea, abdominal cramps, nausea, vomiting, chills, and fever [1]. Less frequently, V. parahaemolyticus infection may lead to cellulitis (or necrotizing fasciitis) with swelling and pain at the site of infection or septicemia with low blood pressure and shock [1]. The major virulence factors expressed by $V$. parahaemolyticus include thermostable direct hemolysin (TDH), TDH-related hemolysin (TRH), type III secretion system (T3SS) 1 and T3SS2 [1].

The membrane fusion protein $(m f p)$ gene locus (VPA1446-1443) consists of two operons cpsQ-mfpABC and $m f p A B C$ [2]. $c p s Q$ encodes a c-di-GMP binding protein that acts as a positive regulator of capsular polysaccharide (cps) genes and $m f p A B C$ transcription [3]. $m f p A$

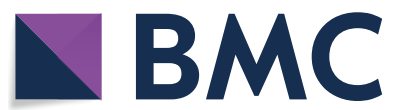

(c) The Author(s) 2021. This article is licensed under a Creative Commons Attribution 4.0 International License, which permits use, sharing, adaptation, distribution and reproduction in any medium or format, as long as you give appropriate credit to the original author(s) and the source, provide a link to the Creative Commons licence, and indicate if changes were made. The images or other third party material in this article are included in the article's Creative Commons licence, unless indicated otherwise in a credit line to the material. If material is not included in the article's Creative Commons licence and your intended use is not permitted by statutory regulation or exceeds the permitted use, you will need to obtain permission directly from the copyright holder. To view a copy of this licence, visit http://creativeco mmons.org/licenses/by/4.0/. The Creative Commons Public Domain Dedication waiver (http://creativecommons.org/publicdomain/ zero/1.0/) applies to the data made available in this article, unless otherwise stated in a credit line to the data. 
encodes a potential secreted calcium-binding protein, $m f p B$ encodes a potential ABC-type transporter, and $m f p C$ encodes a type 1 secretion membrane fusion protein homologous to HlyD [3]. Proteins encoded by the $m f p$ locus are required for biofilm formation by $V$. parahaemolyticus, and $m f p$ mutants have severe defects in biofilm formation and display altered colony morphology and color when grown on Congo red medium [4]. The master quorum sensing (QS) regulators AphA and OpaR oppositely regulate the transcription of cpsQ-mfpABC and $m f p A B C$ during different stages of $V$. parahaemolyticus growth, leading to gradual increases in their transcription with the transition from low cell density (LCD) to high cell density (HCD) [2]. The LysR-type transcriptional regulator CalR is calcium-regulated transcription factor that also can bind to the upstream DNA regions of $m f p A B C$ to activate its transcription $[5,6]$.

In addition to directly regulating $m f p A B C$ transcription, CalR was shown to be involved in directly regulating transcription of the type VI secretion system 2 (T6SS2) gene, $t d h 2$ and T3SS1 genes as well as swarming motility [5-8]. In addition, transcription of calR is directly activated by the transmembrane regulator ToxR, which was first described as a transcriptional activator of cholera toxin $[7,9,10]$. As a feedback of ToxR activation, CalR represses its own transcription and that of toxR in a direct manner [7]. Moreover, ToxR binds to the promoter-proximal DNA regions of T3SS1 genes to repress their transcription, and occupies the regulatory regions of $t$ dh 2 and T3SS2 genes to activate their transcription [10-13]. ToxR is also required for biofilm formation, motility, and stress tolerance of V. parahaemolyticus [10, 14]. Expression of ToxR itself is dependent on cell density in V. parahaemolyticus [11]. However, the master QS regulators AphA and OpaR do not directly regulate tox $R$ transcription [11]. Although autorepression of ToxR may contribute to cell density-dependent transcription [11], there are likely other unknown regulators contributing to this process.

The QS and virulence regulator QsvR, an AraC-type transcriptional protein, was originally described as a repressor of biofilm formation in in $V$. parahaemolyticus, as $q s v R$ mutant formed robust and distinctive puffballshaped biofilms [4]. Recently work demonstrated that expression levels of QsvR were consistent with those of OpaR, and both occurred at HCD [15]. In addition, QsvR directly represses aphA but activates opaR transcription, thereby working with the QS system to tightly regulate the expression of major virulence gene loci such as T3SS1, T3SS2, T6SS2, and tdh2 [15-17]. In order to detect whether QsvR contributes to cell density-dependent transcription of tox $R$, we performed a series of experiments to investigate the regulatory actions of QsvR on tox $R$ transcription. ToxR directly activates the transcription of calR [7], whereas CalR directly activates the transcription of $m f p A B C$ [5]. Therefore, we also detected whether QsvR regulates transcription of both calR and the genes within the $m f p$ locus. The results showed that transcription of toxR, calR, cpsQ-mfpABC, and $m f p A B C$ were under the direct regulation of QsvR. QsvR represses tox $R$ and calR transcription as well as activating the transcription of cpsQ-mfpABC and $m f p A B C$.

\section{Results \\ QsvR represses toxR transcription}

The highest reported levels of tox $R$ transcription are seen at optical density at $600 \mathrm{nM}\left(\mathrm{OD}_{600}\right)$ values between 0.2 and 0.4 in $V$. parahaemolyticus strains grown in completed heart infusion (HI) broth at $37{ }^{\circ} \mathrm{C}$ [11]. However, the master QS regulators AphA and OpaR do not seem to directly regulate toxR transcription [11]. The highest expression levels of QsvR occur at $\mathrm{OD}_{600}$ values between 0.4 and 0.8 , which is similar to OpaR [15]. Additionally, QsvR directly activates opaR transcription [15]. Thus, we decided to assess whether QsvR contributed to cell density-dependent tox $R$ transcription. Bacterial cells were harvested at an $\mathrm{OD}_{600}$ value of 0.8 and analyzed using quantitative real-tome PCR (qPCR) and primer extension assays (Figs. 1a and b). The results showed that mRNA levels of toxR increased significantly in the $\Delta q s v R$ strain compared with the wild-type (WT) strain, suggesting that toxR transcription was negatively regulated by QsvR. The promoter-proximal DNA region of tox $R$ was cloned into the plasmid pHRP309, which contains a promoterless lacZ reporter gene. The recombinant plasmid was then transferred into the $\Delta q s v R$ and WT strains, which were analyzed using LacZ fusion assays. The results showed that the promoter activity of toxR was much higher in the $\Delta q s v R$ strain than that in the WT strain, suggesting that the promoter activity of tox $R$ was negatively regulated by QsvR (Fig. 1c). The promoter-proximal DNA region of toxR was obtained by PCR, and then subjected to electrophoretic mobility shift assay (EMSA). The results showed that His-QsvR was able to specifically bind to the promoter-proximal DNA fragment of toxR in a dose-dependent manner in vitro (Fig. 1d). A DNase I footprinting assay was then employed to detect the QsvR binding sites within the upstream DNA fragment of toxR. As shown in Fig. 1e, His-QsvR protected a single DNA region from 179 to $43 \mathrm{bp}$ upstream of toxR against DNase I digestion. Taken together, these results suggested that QsvR directly repressed the transcription of toxR.

\section{QsvR represses calR transcription}

A previous study demonstrated that ToxR specifically bound to the promoter-proximal DNA region of calR 


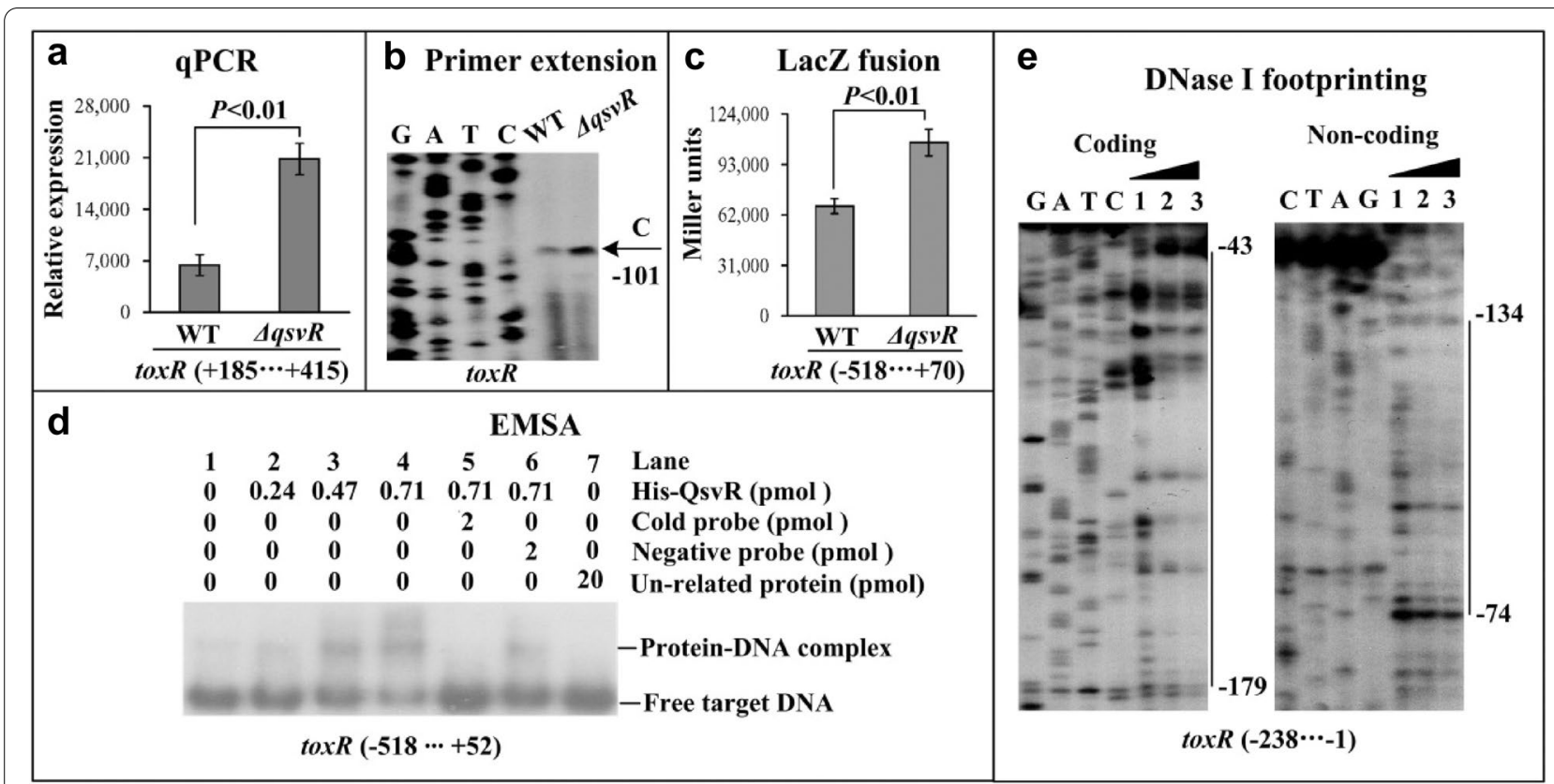

Fig. 1 Regulation of toxR by QsvR. V. parahaemolyticus strains were grown in $\mathrm{HI}$ broth at $37^{\circ} \mathrm{C}$, and bacterial cells were harvested at an $\mathrm{OD}_{600}$ value of 0.8 . Negative and positive numbers indicate the nucleotide positions upstream and downstream of toxR, respectively. a qPCR. Relative mRNA levels of toxR were tested in WT and $\triangle q S v R$ strains. $\mathbf{b}$ Primer extension. An oligonucleotide primer complementary to the toxR RNA transcript was designed. The primer extension products were analyzed with an $8 \mathrm{M}$ urea- $6 \%$ acrylamide sequencing gel. $\mathbf{c}$ LacZ fusion. The promoter-proximal DNA region of toxR was cloned into the pHRP309 plasmid and then transferred into WT and $\triangle q$ svR strains to determine promoter activity (Miller units) in the cellular extracts. $\mathbf{d}$ EMSA. The radioactively-labelled promoter-proximal DNA fragments of tox $R$ were incubated with increasing amounts of His-QsvR and analyzed using 4\% (w/v) polyacrylamide gel electrophoresis. e DNase I footprinting. Labelled coding or noncoding DNA probes were incubated with increasing amounts of His-QsvR and analyzed using DNase I footprinting. The footprint regions are indicated by vertical bars at the corresponding sequence positions. Lanes C, T, A and G represent Sanger sequencing reactions

to activate its transcription [7]. The direct repression of toxR transcription by QsvR indicated that the transcription of calR might be also regulated by QsvR in $V$. parahaemolyticus. Therefore, we employed $\mathrm{qPCR}$ and primer extension assays to test QsvR-dependent transcription of calR. The results showed that mRNA levels of calR increased significantly in the $\Delta q s v R$ strain compared to the WT strain, indicating that transcription of calR was negatively regulated by QsvR (Figs. 2a and $b$ ). The result of LacZ fusion assays showed that the promoter activity of $c a l R$ was significantly enhanced in the $\Delta q s v R$ strain compared to the WT strain (Fig. 2c). The result of the EMSA demonstrated that His-QsvR was able to specifically bind to the promoter-proximal DNA fragments of calR in a dose-dependent manner (Fig. 2d). The DNase I footprinting assay further detected a single His-QsvR binding site located from 175 to $45 \mathrm{bp}$ upstream of calR. Thus, the transcription of calR was directly repressed by QsvR in $V$. parahaemolyticus.

\section{QsvR activates the transcription of $c p s Q-m f p A B C$ and $m f p A B C$}

CalR directly activates the transcription of $m f p A B C$ but indirectly activates $c p s Q-m f p A B C$ transcription [5]. Thus, the direct regulation of calR by QsvR promoted us to detect whether QsvR has regulatory actions on the transcription of $c p s Q-m f p A B C$ and $m f p A B C$ in V.parahaemolyticus. qPCR (Fig. 3a) and primer extension (Fig. 3b) assays were carried out to measure mRNA levels of $c p s Q$ and $m f p A$ in $\Delta q s v R$ and WT strains. The results showed that mRNA levels of both genes were significantly lower in the $\Delta q s v R$ strain compared to the WT strain. The LacZ fusion assay demonstrated that the promoter activities of $c p s Q$ and $m f p A$ were also significantly lower in the $\triangle q s v R$ strain compared to the WT strain (Fig. 3c). In vitro EMSA results showed that His-QsvR was able to specifically bind to the promoter-proximal DNA fragments of both cpsQ and cpsA in a dose-dependent manner (Fig. 3d). As further determined by DNase I footprinting assay (Fig. 3e), His-QsvR protected a single DNA region 


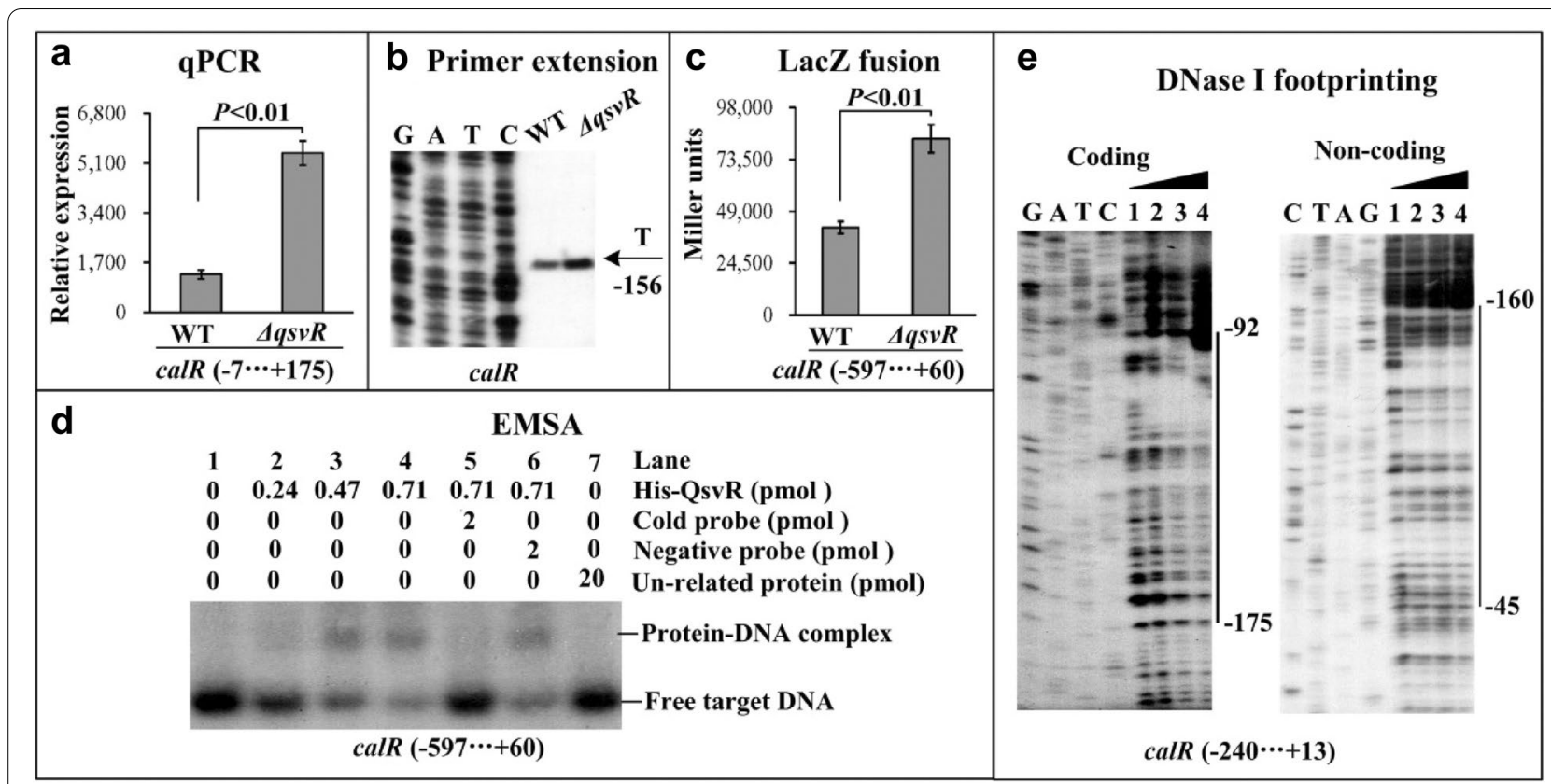

Fig. 2 Regulation of calR by QsvR. Negative and positive numbers indicate the nucleotide positions upstream and downstream of calR, respectively. Lanes C, T, A and G represent Sanger sequencing reactions. The GPCR (a), primer extension (b), LacZ fusion (c), EMSA (d) and DNase I footprinting (e) were performed as described in Fig. 1

for each of the promoter-proximal DNA region of cpsQ and $m f p A$ against DNase I digestion, located from 156 to 19 bp upstream of $c p s Q$ and from 231 to 65 bp upstream of $m f p A$. Taken together, these results demonstrated that QsvR directly activated the transcription of both cpsQ$m f p A B C$ and $m f p A B C$ in $V$. parahaemolyticus.

ToxR exerts no regulatory action on the promoter activities of $c p s Q-m f p A B C$ or $m f p A B C$

Bacterial cells were harvested at an $\mathrm{OD}_{600}$ value of 0.4 , and then subjected to the qPCR and LacZ fusion assays to investigate ToxR-mediated cpsQ-mfpABC and $m f p A B C$ transcription [11]. As shown in Fig. 4, mRNA levels of both cps $Q$ and $m f p A$ were similar in $\triangle t o x R$ and WT strains, and $\beta$-galactosidase activity in the cellular extracts of cpsQ and $m f p A$ were similar to those of the $\triangle$ toxR and WT strains. These results suggested that ToxR had no regulatory effect on the transcription of cpsQ$m f p A B C$ or $m f p A B C$.

\section{Discussion}

High transcriptional levels of toxR were observed in $V$. parahaemolyticus when the bacteria was grown in $\mathrm{HI}$ broth and harvested at $\mathrm{OD}_{600}$ values between 0.2 and 0.4 [11]. However, the reasons for this phenomenon are still not full understood. The data presented here showed that QsvR bound to the promoter-proximal DNA region of toxR to repress its transcription in bacterial cells harvested during the mid-logarithmic growth phase. Negative autoregulation of ToxR at LCD (or during the LCD-to-HCD transition) and repression of tox $R$ by CalR during the mid-logarithmic growth phase have previously been demonstrated in $V$. parahaemolyticus $[7,11]$. Thus, we hypothesized that the cell density-dependent transcription of toxR was likely due to the synergistic and sequential regulation of ToxR, CalR and QsvR throughout the growth cycle. At LCD (or during the LCD-toHCD transition), ToxR binds to its own promoter to repress its own gene transcription via direct interference with the action of RNA polymerase (RNAP) [11]. At HCD, the bacterium replaced ToxR with CalR and QsvR, leading to repression of tox $R$ transcription. The binding sites of CalR and QsvR overlap each other as well as the -35 and -10 elements and transcription start site of toxR (Fig. 5a). Thus, QsvR may work with CalR to silence the transcription of toxR by directly interfering with RNAP action.

Direct activation of calR by ToxR and feedback repression of tox $R$ by CalR have previously been reported [7]. The direct repression of toxR by QsvR reported in the present study promoted us to detect whether QsvR regulated calR transcription. The data showed that QsvR could bind to the promoter-proximal DNA region of calR to repress its transcription. The binding site of QsvR for calR overlaps with the CalR site as well as the -35 and -10 elements and transcription start site of calR (Fig. 5b). 


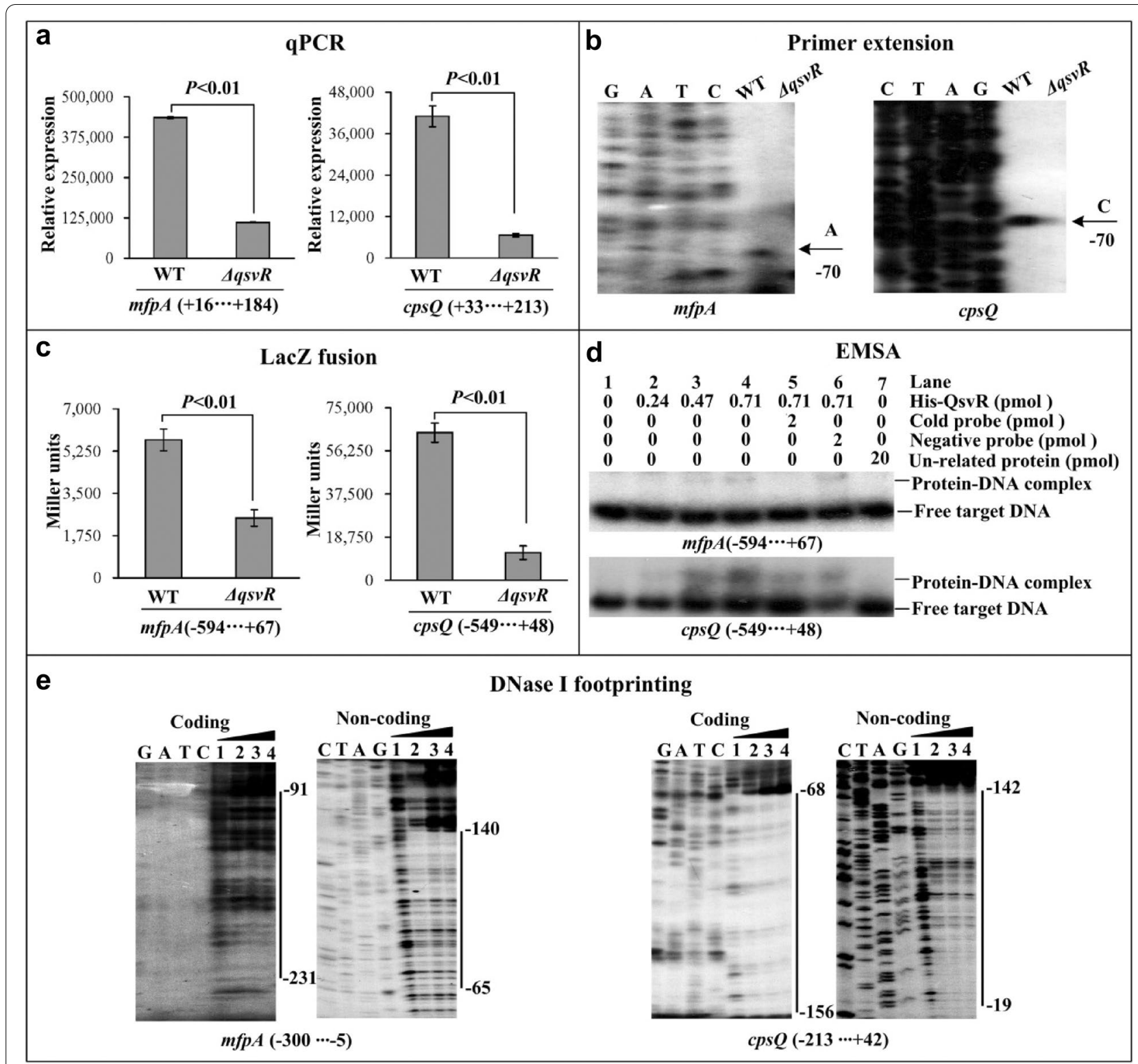

Fig. 3 Regulation of $c p s Q-m f p A B C$ and $m f p A B C$ by QsvR. Negative and positive numbers indicate the nucleotide positions upstream and downstream of cpsQ-mfpABC and $m f p A B C$, respectively. Lanes $C, T, A$ and $G$ represent Sanger sequencing reactions. The qPCR (a), primer extension (b), LacZ fusion (c), EMSA (d) and DNase I footprinting (e) were performed as described in Fig. 1

Thus, QsvR may also work with CalR to silence the transcription of calR by directly interfering with RNAP action. ToxR activation of calR transcription belongs to class I transcriptional stimulation, as its binding site is far from the -35 element $[7,18]$. Direct regulation of calR and toxR transcription by QsvR indicated that QsvR controlled all of the genes within the CalR and ToxR regulons in V. parahaemolyticus.

The data presented here also showed that QsvR bound to the promoter-proximal DNA regions of cps $Q-m f p A B C$ and $m f p A B C$ to activate their transcription. The binding sites of QsvR for cpsQ-mfpABC and $m f p A B C$ also overlap with their -35 and -10 elements and transcription start sites (Figs. $5 \mathrm{c}$ and d). This is an abnormal mechanism for a transcriptional regulator to activate transcription of its target genes. However, this phenomenon is expected because similar QsvR-dependent promoters have been found in V. parahaemolyticus [15]. There are likely additional unknown regulators that repress the transcription of $m f p$ genes in V. parahaemolyticus at the same growth 


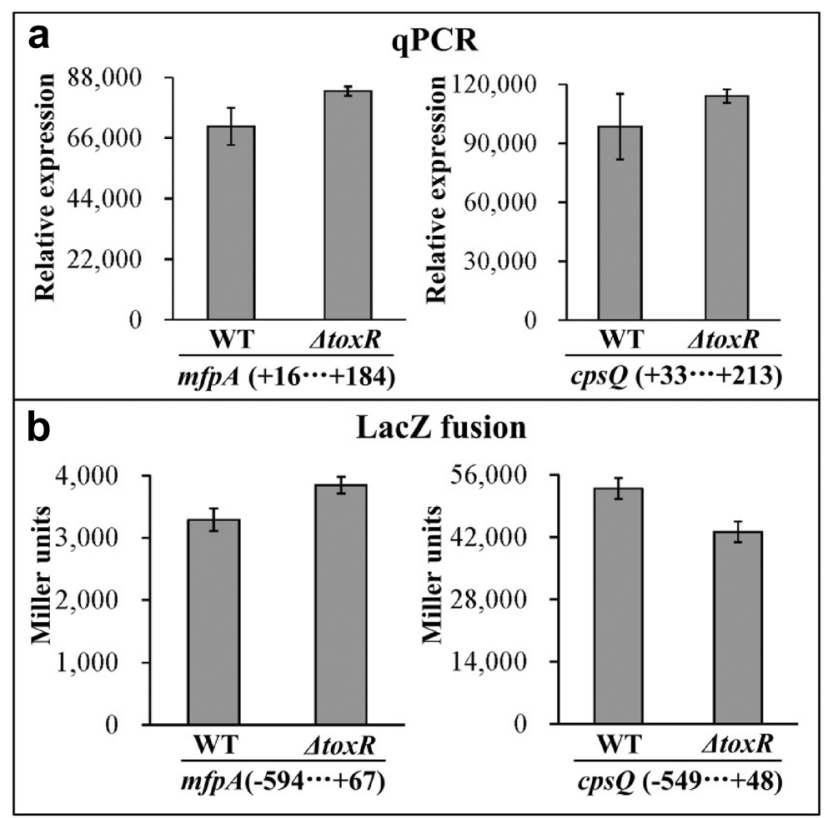

Fig. 4 Regulation of $c p s Q-m f p A B C$ and $m f p A B C$ by ToxR. V. parahaemolyticus strains were grown in $\mathrm{HI}$ broth at $37^{\circ} \mathrm{C}$, and bacterial cells were harvested at an $\mathrm{OD}_{600}$ value of 0.4. The $\mathrm{qPCR}(\mathbf{a})$ and LacZ fusion (b) were performed as described in Fig. 1

conditions. QsvR may antagonizer these repressors, leading to the activation of transcription of genes within the $m f p$ locus [8]. Both CalR and OpaR have been reported to be required for the expression of the $m f p$ locus [2, 5], whereas transcription of calR and opaR is under the direct control of QsvR according to data presented here and in the Ref. [15]. Because QsvR directly activates $o p a R$ transciption, deletion of $q s v R$ should also result in reduced expression of OpaR, which may further reduce the expression of genes within the $m f p$ locus. The data presented here and in previous studies led us to suggest a complex regulatory circuit involving co-regulation of the $m f p$ locus by QsvR, OpaR, CalR, and ToxR (Fig. 6), which contributed to a deeper understanding of the regulatory network of the $m f p$ locus in $V$. parahaemolyticus. Although the detailed molecular mechanisms are still unclear, both CpsQ and MfpABC have been reported to be required for biofilm formation in $V$. parahaemolyticus $[3,4]$. The regulation of $c p s Q-m f p A B C$ and $m f p A B C$ transcription by QsvR, OpaR and ToxR may be one of the mechanisms regulating biofilm formation in $V$. parahaemolyticus.

\section{Conclusion}

QsvR directly repressed the transcription of toxR and calR, whereas it directly activated cpsQ-mfpABC and $m f p A B C$ transcription. Our results on the regulation of toxR, calR, cps $Q-m f p A B C$ and $m f p A B C$ by QsvR provided us with deeper understanding of the regulatory network of the $m f p$ locus in V. parahaemolyticus.

\section{Materials and methods \\ Bacterial strains}

V. parahaemolyticus strain RIMD2210633 (wild type, WT) was used in the current study [19]. Nonpolar $q s v R$ and tox $R$ single-gene deletion mutants $(\Delta q s v R$ and $\Delta t o x R)$ derived from the WT strain were described in our previous studies $[11,15]$. All primers used in this study are listed in Table 1.

\section{Bacterial growth conditions}

Vibrio parahaemolyticus strains were grown in 2.5\% Bacto heart infusion (HI; BD Bioscience, USA) broth at $37{ }^{\circ} \mathrm{C}$ with shaking at $250 \mathrm{r} / \mathrm{min}$ [15]. Briefly, overnight bacterial cultures were diluted 50 -fold into $15 \mathrm{ml}$ of fresh $\mathrm{HI}$ broth, and were allowed to grow at $37^{\circ} \mathrm{C}$ to $\mathrm{OD}_{600} \approx 1.0$ (mid-exponential growth phase). Thereafter, the cultures were diluted 1000-fold into $15 \mathrm{ml}$ of fresh $\mathrm{HI}$ broth for a third round of cultivation. Bacterial cells were harvested at the required cell densities. When necessary, the medium was supplemented with $50 \mu \mathrm{g} / \mathrm{ml}$ gentamicin.

\section{Quantitative real-time PCR (qPCR)}

The qPCR assay was performed as previously described [20]. Briefly, total RNAs were extracted from $V$. parahaemolyticus strains using the TRIzol Reagent 


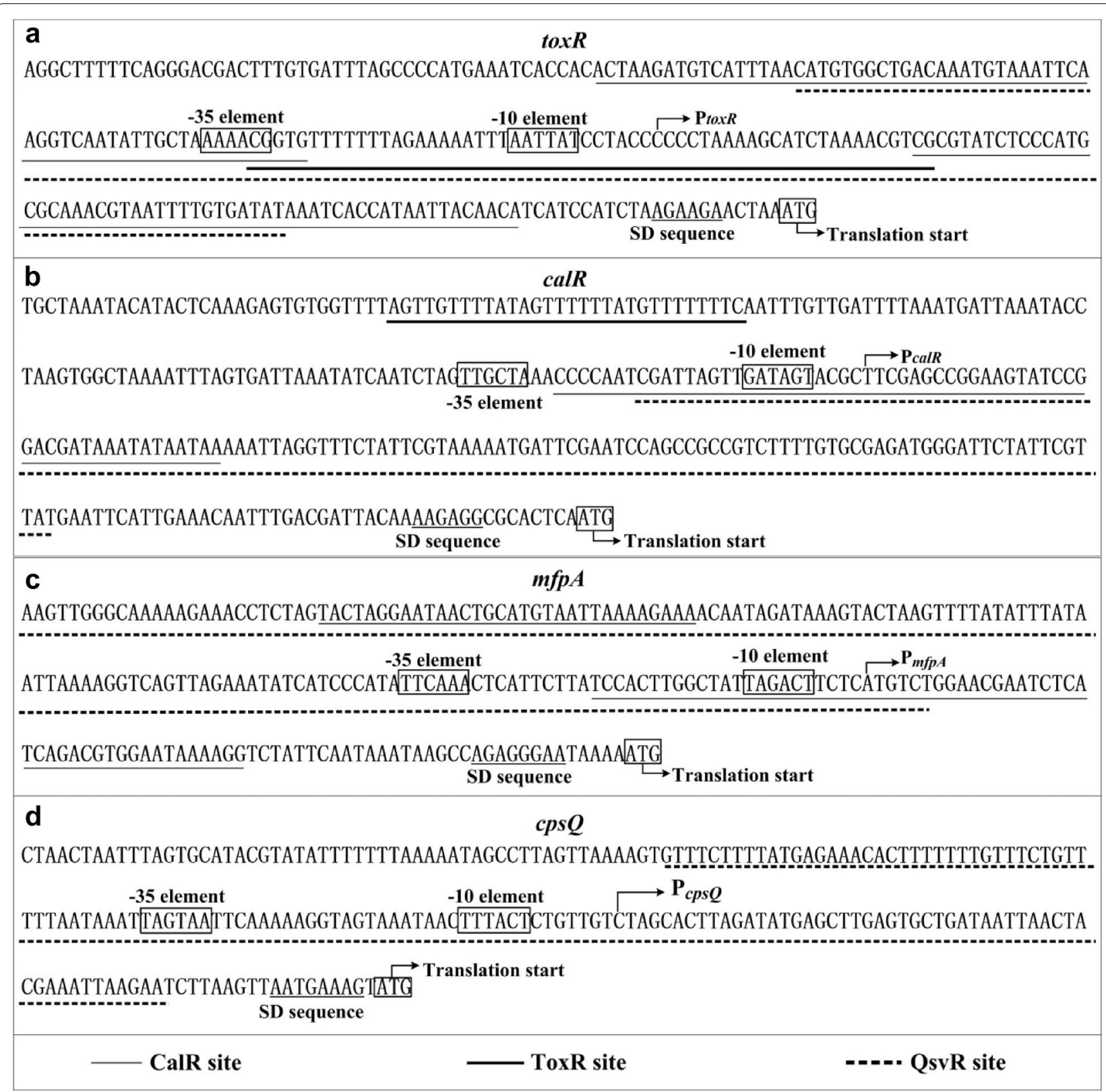

Fig. 5 Promoter structure of target genes. The promoter DNA regions of indicated genes were derived from RIMD 2210633. The translation and transcription starts are shown with bent arrows. The predicted core promoter -10 and -35 elements and the SD sequences are boxed. The binding sites of regulators are underlined

(Invitrogen, USA). Contaminated DNA in the total RNAs was removed using an Ambion's DNA-free ${ }^{\mathrm{TM}}$ Kit (Ambion Inc., USA) according to the manufacturer's instructions. cDNA was generated using $8 \mu \mathrm{g}$ of total RNAs and $3 \mu \mathrm{g}$ of random hexamer primers. The relative mRNA levels of each target gene were determined based on a standard curve of $16 \mathrm{~S}$ rRNA (reference gene) expression performed for each RNA preparation. The annealing condition for all primer pairs was $54{ }^{\circ} \mathrm{C}$ for $4 \mathrm{~s}$.

\section{Primer extension assay}

Primer extension assay was performed as previously described [20, 21]. Briefly, approximately $10 \mu \mathrm{g}$ of total RNAs were annealed with $1 \mathrm{pmol}$ of $5^{\prime}-{ }^{32} \mathrm{P}$-end labelled reverse primer to generate cDNAs using a Primer Extension System (Promega, USA). The same labelled primer was also used for sequencing with the AccuPower \& Top DNA Sequencing Kit (Bioneer, Korea) according to the manufacturer's instructions. The products of primer 


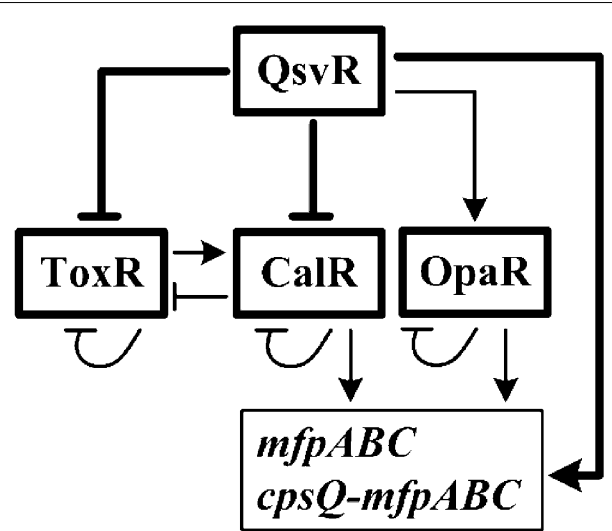

Fig. 6 Regulatory circuit. Arrows represent positive regulation, and vertical lines represent negative regulation. Elements described in previous studies are indicated with thin lines, and those described in the current study are indicated with bold lines

extension and sequencing were concentrated and analyzed in an $8 \mathrm{M}$ urea- $6 \%$ polyacrylamide gel electrophoresis, and the results were detected by autoradiography using Fuji Medical X-ray film (Fuji Photo Film Co., Ltd. Japan).

\section{LacZ fusion and $\beta$-galactosidase assay}

The promoter-proximal DNA region of each target gene was cloned into the corresponding restriction endonuclease sites of pHRP309, which harbors a promoterless lac $Z$ reporter gene and a gentamicin resistance gene [22]. The recombinant plasmid was subsequently transferred into the WT strain and the deletion mutants to measure the $\beta$-galactosidase activities of the cellular extracts using a $\beta$-Galactosidase Enzyme Assay System (Promega, USA) according to the manufacturer's instructions [15, 20]. Briefly, the assay was performed by adding $30 \mu \mathrm{L}$ of diluted sample to an equal volume of assay $2 \times$ buffer that containing the substrate $o$-nitrophenyl- $\beta$-Dgalactopyranoside. Samples were incubated for approximately $30 \mathrm{~min}$, during which time the $\beta$-galactosidase hydrolyzes the colorless substrate to $o$-nitrophenol, which is yellow. The reaction was terminated by the addition of $90 \mu \mathrm{L}$ sodium carbonate, and the absorbance was read at $\mathrm{OD}_{420}$ and $\mathrm{OD}_{550}$ with a spectrophotometer. The number of Miller units (representing the galactosidase activity) was calculated using the following formula: $10^{6} \times\left[\left(\mathrm{OD}_{420}-1.75 \times \mathrm{OD}_{550}\right) /\left(\mathrm{T} \times \mathrm{V} \times \mathrm{OD}_{600}\right)\right] . \quad$ The Miller units represent the change in the $\mathrm{OD}_{420} / \mathrm{min} / \mathrm{ml}$ relative to the $\mathrm{OD}_{600}$ of the cells.

\section{Preparation of $6 \times$ His-tagged QsvR (His-QsvR)}

The entire coding region of $q s v R$ was cloned into the corresponding restriction endonuclease sites of pET28a plasmid (Novagen, USA). Thereafter, the recombinant plasmid encoding His-QsvR was then transferred into E. coli BL21入DE3 for protein expression [23]. Expression and purification of His-QsvR was performed in a manner similar to that described previously for His-OpaR [21].

\section{Electrophoretic mobility shift assay (EMSA)}

EMSA was carried out as previously described [15, 21]. Briefly, the 5'-ends of the promoter-proximal DNA region of each target gene were labelled with $\left[\gamma_{-}{ }^{32} \mathrm{P}\right]$ ATP using the T4 polynucleotide kinase. EMSA was performed in a $10 \mu \mathrm{l}$ reaction volume containing binding buffer $(1 \mathrm{mM} \mathrm{MgCl} 2,0.5 \mathrm{mM}$ EDTA, $0.5 \mathrm{mM}$ DTT, $50 \mathrm{mM} \mathrm{NaCl}, 10 \mathrm{mM}$ Tris- $\mathrm{HCl} / \mathrm{pH} 7.5$ and $10 \mathrm{mg} / \mathrm{ml}$ salmon sperm DNA), labelled DNA probe, and increasing amounts of His-QsvR. Three controls were included in each EMSA experiment: (1) cold probe as a specific DNA competitor (the same unlabeled DNA fragments), (2) negative probe as a non-specific DNA competitor (the unlabeled coding region of the $16 \mathrm{~S}$ rRNA gene) and (3) non-specific protein competitor (rabbit anti-F1-protein polyclonal antibodies). The products were analyzed in a native $4 \%(\mathrm{w} / \mathrm{v})$ polyacrylamide gel, and the results were detected by autoradiography after exposure to Fuji Medical X-ray film.

\section{DNase I footprinting}

The DNase I footprinting assay was performed as previously described $[15,21]$. Briefly, DNA binding was carried out in $10 \mu \mathrm{l}$ reaction containing binding buffer, single strand $5^{\prime}$-ends ${ }^{32} \mathrm{P}$-labelled probe, and increasing amounts of His-QsvR and incubated at room temperature for $30 \mathrm{~min}$. Before digestion, $10 \mu \mathrm{l}$ of $\mathrm{Ca}^{2+} / \mathrm{Mg}^{2+}$ solution $\left(5 \mathrm{mM} \mathrm{CaCl}_{2}\right.$ and $10 \mathrm{mM} \mathrm{MgCl}$ ) was added to each reaction and incubated at at room temperature for 1 min. Optimized RQ1 RNase-Free DNase I (Promega, USA) was added to each reaction mixture, and the mixture was incubated at room temperature for 40-90 s. The reaction was quenched by adding $9 \mu \mathrm{l}$ of stop solution (200 mM NaCl, $30 \mathrm{mM}$ EDTA, and 1\% SDS). The partially digested DNA samples were extracted with phenol/ chloroform, precipitated with ethanol, and analyzed in $6 \%$ polyacrylamide/8 M urea gels. Protected regions were identified by comparison with sequence ladders. The templates used for DNA sequencing were the same as the DNA fragments used in the DNase I footprinting assays. The results were detected by autoradiography after exposure to Fuji Medical X-ray film.

\section{Experimental replicates and statistical methods}

At least three independent replicates of the LacZ fusion and $\mathrm{qPCR}$ assays were performed, and primer extension, EMSA, and DNase I footprinting assays were 


\section{Table 1 Oligonucleotide primers used in this study}

\begin{tabular}{|c|c|}
\hline Target & Primers (forward/reverse, $5^{\prime}-3^{\prime}$ ) \\
\hline \multicolumn{2}{|c|}{ Construction of mutants } \\
\hline \multirow[t]{3}{*}{$q s \vee R$} & GTGACTGCAGATGCTAAAAGCGGTGATTC/GATTCAAATGCGATTTCTGTTGGCTGGTGGACGACTAATG \\
\hline & CATTAGTCGTCCACCAGCCAACAGAAATCGCATTTGAATC/GTGAGCATGCGAGAAGTCTGTAAACGAAACG \\
\hline & GTGACTGCAGATGCTAAAAGCGGTGATTC/GTGAGCATGCGAGAAGTCTGTAAACGAAACG \\
\hline \multirow[t]{3}{*}{ toxR } & GTGACTGCAGAAACGCAATTTGTCTGATG/ATCTTCATGCTGGCCTCCTTTAGTTCTTCTTAGATGGATGATG \\
\hline & CATCATCCATCTAAGAAGAACTAAAGGAGGCCAGCATGAAGAT/GTGAGCATGCAATTCGGCGGCTTTGTTC \\
\hline & GTGACTGCAGAAACGCAATTTGTCTGATG/GTGAGCATGCAATTCGGCGGCTTTGTTC \\
\hline \multicolumn{2}{|c|}{ Protein expression } \\
\hline$q s \vee R$ & AGCGGGATCCATGCCGAACATTGAGATCATTC/AGCGAAGCTTTTAACCTCTTACTACCTGATTACG \\
\hline \multicolumn{2}{|l|}{ qPCR } \\
\hline toxR & TTGTTTGGCGTGAGCAAGG/TAGCAGAGGCGTCATTGTTATC \\
\hline calR & ATGTAAAAAGAAAACCGTACA/AACACAGCAGAATGACCGTG \\
\hline cpsQ & GCCTGAAATCCTAATGCTC/AGTGTCAGAAGGTGTATCAAC \\
\hline$m f p A$ & GCGGGCAATGATCGTCTAAC/TCACCTGAACCTGCGACAAG \\
\hline \multicolumn{2}{|c|}{ Primer extension } \\
\hline toxR & /TTAGTTCTTCTTAGATGGATGATG \\
\hline calR & /GCAAAATATCGGTACTTCA \\
\hline $\operatorname{cps} Q$ & /GATTTCAGGCTTTTCCGTGTAC \\
\hline$m f p A$ & /ATTCCCTCTGGCTTATTTATTG \\
\hline \multicolumn{2}{|c|}{ LacZ fusion } \\
\hline tox $R$ & GCGCGTCGACATCGTTAAGGTATTTGCA/GCGCGAATTCCGAGCGAATTACTATTTGG \\
\hline calR & GCGGTCGACGTTTGTTTGCTCGGATTGTTTG/GCGTCTAGACAAAGTGCTTTCCATACGGTAG \\
\hline $\operatorname{cps} Q$ & GCGCGTCGACCAGACGGGCATTGATAAG/GCGCGAATTCCATTAGGATTTCAGGCTTTT \\
\hline$m f p A$ & GCGCGTCGACTTATGACTTAGATACCGAA/GCGCGAATTCCGAAATCAGCGATATTGTTG \\
\hline \multicolumn{2}{|l|}{ EMSA } \\
\hline toxR & ATCGTTAAGGTATTTGCA/CGAGCGAATTACTATTTGG \\
\hline calR & GTTTGTTTGCTCGGATTGTTTG/CAAAGTGCTTTCCATACGGTAG \\
\hline cpsQ & GTTCCAGCAATACTGACTAAGC/GATTTCAGGCTTTTCCGTGTAC \\
\hline$m f p A$ & TAGGACGCAAGCCACAAG/CGAAATCAGCGATATTGTTG \\
\hline \multicolumn{2}{|c|}{ DNase I footprinting } \\
\hline toxR & TTTCAGGGACGACTTTGTG/TTAGTTCTTCTTAGATGGATGATG \\
\hline calR & ATTCCCTCTGGCTTATTTATTG/CCACGGCATTACTTACTG \\
\hline $\operatorname{cps} Q$ & TACCTAACTAATTTAGTGCA/GATTTCAGGCTTTTCCGTGTAC \\
\hline$m f p A$ & ACATACTATTAAATCGCATC/ATTCCCTCTGGCTTATTTATTG \\
\hline
\end{tabular}

each performed at least twice. Values are expressed as mean \pm standard deviation (SD). Paired Student's $t$-tests were used to calculate statistically significant differences, and $P$ values $<0.01$ were considered statistically significant.

\section{Authors' contributions}

$Y Z, D Z$, and RL designed, organized, and supervised the experiments, interpreted the results, and edited the manuscript. $Y Z, Y Q, X X, M Z$, JS, XL, HL, ZY and WY performed laboratory experiments. YZ drafted the manuscript. All authors read and approved the final manuscript.

\section{Funding}

This work was funded by the National Natural Science Foundation of China (no. 82072239), the Jiangsu Planned Projects for Postdoctoral Research
Funds (no. 2020Z102), and the China Postdoctoral Science Foundation (no. 2020M681513).

\section{Data availability}

Not applicable.

\section{Ethics approval and consent to participate}

Not applicable.

\section{Consent for publication}

Not applicable.

\section{Competing interests}

Not applicable.

\author{
Author details \\ ${ }^{1}$ Wuxi School of Medicine, Jiangnan University, Wuxi 214122, Jiangsu, \\ China. ${ }^{2}$ School of Medicine, Jiangsu University, Zhenjiang 212013, Jiangsu,
}


China. ${ }^{3}$ State Key Laboratory of Pathogen and Biosecurity, Beijing Institute of Microbiology and Epidemiology, Beijing 100071, China. ${ }^{4}$ Department of Clinical Laboratory, Nantong Third Hospital Affiliated to Nantong University, Nantong 212006, Jiangsu, China.

Received: 6 December 2020 Accepted: 23 February 2021 Published online: 02 March 2021

\section{References}

1. Broberg CA, Calder TJ, Orth K. Vibrio parahaemolyticus cell biology and pathogenicity determinants. Microbes Infect. 2011;13(12-13):992-1001. https://doi.org/10.1016/j.micinf.2011.06.013.

2. Zhou D, Yan X, Qu F, Wang L, Zhang Y, Hou J, et al. Quorum sensing modulates transcription of cpsQ-mfpABC and mfpABC in Vibrio parahaemolyticus. Int J Food Microbiol. 2013;166(3):458-63. https://doi.org/10.1016/j. ijfoodmicro.2013.07.008.

3. Ferreira RB, Chodur DM, Antunes LC, Trimble MJ, McCarter LL. Output targets and transcriptional regulation by a cyclic dimeric GMPresponsive circuit in the Vibrio parahaemolyticus Scr network. J Bacteriol. 2012;194(5):914-24. https://doi.org/10.1128/JB.05807-11.

4. Enos-Berlage JL, Guvener ZT, Keenan CE, McCarter LL. Genetic determinants of biofilm development of opaque and translucent Vibrio parahaemolyticus. Mol Microbiol. 2005;55(4):1 160-82. https://doi.org/10.1 111/j.1365-2958.2004.04453.x.

5. Gao H, Zhang L, Osei-Adjei G, Yang W, Zhou D, Huang X, et al. Transcriptional regulation of cpsQ-mfpABC and $\mathrm{mfpABC}$ by CalR in Vibrio parahaemolyticus. MicrobiologyOpen. 2017. https://doi.org/10.1002/ mbo3.470.

6. Gode-Potratz CJ, Chodur DM, MCCarter LL. Calcium and iron regulate swarming and type III secretion in Vibrio parahaemolyticus. J Bacteriol. 2010;192(22):6025-38. https://doi.org/10.1128/JB.00654-10.

7. Osei-Adjei G, Gao H, Zhang Y, Zhang L, Yang W, Yang H, et al. Regulatory actions of ToxR and CalR on their own genes and type III secretion system 1 in Vibrio parahaemolyticus. Oncotarget. 2017;8(39):65809-22. https:// doi.org/10.18632/oncotarget.19498.

8. Zhang L, Osei-Adjei G, Zhang Y, Gao H, Yang W, Zhou D, et al. CalR is required for the expression of T6SS2 and the adhesion of Vibrio parahaemolyticus to HeLa cells. Arch Microbiol. 2017;199(6):931-8. https://doi. org/10.1007/s00203-017-1361-6.

9. Miller VL, Mekalanos JJ. Synthesis of cholera toxin is positively regulated at the transcriptional level by toxR. Proc Natl Acad Sci USA. 1984:81(11):3471-5. https://doi.org/10.1073/pnas.81.11.3471.

10. Whitaker WB, Parent MA, Boyd A, Richards GP, Boyd EF. The Vibrio parahaemolyticus ToxRS regulator is required for stress tolerance and colonization in a novel orogastric streptomycin-induced adult murine model. Infect Immun. 2012;80(5):1834-45. https://doi.org/10.1128/IAI.06284-11.

11. Zhang Y, Hu L, Osei-Adjei G, Yang W, Yin Z, Lu R, et al. Autoregulation of ToxR and its regulatory actions on major virulence gene loci in Vibrio parahaemolyticus. Front Cell Infect Microbiol. 2018;8:291. https://doi. org/10.3389/fcimb.2018.00291.

12. Lin Z, Kumagai K, Baba K, Mekalanos JJ, Nishibuchi M. Vibrio parahaemolyticus has a homolog of the Vibrio cholerae toxRS operon that mediates environmentally induced regulation of the thermostable direct hemolysin gene. J Bacteriol. 1993;175(12):3844-55. https://doi.org/10.1128/ jb.175.12.3844-3855.1993.

13. Hubbard TP, Chao MC, Abel S, Blondel CJ, AbelZurWiesch P, Zhou X, et al. Genetic analysis of Vibrio parahaemolyticus intestinal colonization. Proc Natl Acad Sci USA. 2016;113(22):6283-8.

14. Chen L, Qiu Y, Tang H, Hu LF, Yang WH, Zhu XJ, et al. ToxR is required for biofilm formation and motility of Vibrio parahaemolyticus. Biomed Environ Sci. 2018;31(11):848-50. https://doi.org/10.3967/bes2018.112.

15. Zhang Y, Hu L, Qiu Y, Osei-Adjei G, Tang H, Zhang R, et al. QsvR integrates into quorum sensing circuit to control Vibrio parahaemolyticus virulence. Environ Microbiol. 2019. https://doi.org/10.1111/1462-2920.14524.

16. Qiu Y, Hu L, Yang W, Yin Z, Zhou D, Yang H, et al. The type VI secretion system 2 of Vibrio parahaemolyticus is regulated by QsvR. Microb Pathog. 2020;149:104579. https://doi.org/10.1016/j.micpath.2020.104579.

17. Wang L, Zhou D, Mao P, Zhang Y, Hou J, Hu Y, et al. Cell density- and quorum sensing-dependent expression of type VI secretion system 2 in Vibrio parahaemolyticus. PLoS ONE. 2013;8(8):e73363. https://doi.org/10.1371/ journal.pone.0073363.

18. Ishihama A. Functional modulation of Escherichia coli RNA polymerase. Annu Rev Microbiol. 2000;54:499-518. https://doi.org/10.1146/annur ev.micro.54.1.499.

19. Makino K, Oshima K, Kurokawa K, Yokoyama K, Uda T, Tagomori K, et al. Genome sequence of Vibrio parahaemolyticus: a pathogenic mechanism distinct from that of $\mathrm{V}$ cholerae. Lancet. 2003;361(9359):743-9. https:// doi.org/10.1016/S0140-6736(03)12659-1.

20. Gao H, Zhang Y, Yang L, Liu X, Guo Z, Tan Y, et al. Regulatory effects of CAMP receptor protein (CRP) on porin genes and its own gene in Yersinia pestis. BMC Microbiol. 2011;11:40. https://doi. org/10.1186/1471-2180-11-40.

21. Yu Y, Yang H, Li J, Zhang P, Wu B, Zhu B, et al. Putative type VI secretion systems of Vibrio parahaemolyticus contribute to adhesion to cultured cell monolayers. Arch Microbiol. 2012;194(10):827-35. https://doi. org/10.1007/s00203-012-0816-z.

22. Parales RE, Harwood CS. Construction and use of a new broad-host-range lacZ transcriptional fusion vector, pHRP309, for gram- bacteria. Gene. 1993;133(1):23-30.

23. Kleber-Janke T, Becker WM. Use of modified BL21(DE3) Escherichia coli cells for high-level expression of recombinant peanut allergens affected by poor codon usage. Protein Expr Purif. 2000;19(3):419-24. https://doi. org/10.1006/prep.2000.1265.

\section{Publisher's Note}

Springer Nature remains neutral with regard to jurisdictional claims in published maps and institutional affiliations.

\footnotetext{
Ready to submit your research? Choose BMC and benefit from:

- fast, convenient online submission

- thorough peer review by experienced researchers in your field

- rapid publication on acceptance

- support for research data, including large and complex data types

- gold Open Access which fosters wider collaboration and increased citations

- maximum visibility for your research: over $100 \mathrm{M}$ website views per year
}

At BMC, research is always in progress.

Learn more biomedcentral.com/submissions 